\title{
SHEAR BOND STRENGTH OF A MULTI-MODE UNIVERSAL ADHESIVE CONTAINING HYDROXY-APATITE NANOPARTICLES TO DENTIN (IN VITRO STUDY)
}

\author{
Ahmed K. Ghyadh*, Hala Ragab ${ }^{* *}$ and Essam Osman ${ }^{* * *}$
}

\begin{abstract}
Aim: To evaluate the effect of addition of Hydroxyl-Apatite nanoparticles to a commercial universal adhesive on sheer-bond-strength to dentin using two application modes, immediately and after thermocycling.

Methods: A-commercial UA was used for bonding composite-resin to dentin and considered as control. $10 \%$ of HAp were prepared and added to the commercial UA and considered as experimental. 88-freshly extracted sound human premolar teeth were sectioned to expose midcoronal-dentin. Samples were y divided into: control and experimental group. Each group was further divided based on the application protocol. Half of the samples were stored in distilled water for $24 \mathrm{~h}$ at $37^{\circ} \mathrm{C}$ for immediate SBS testing whereas the other half was subjected to thermocycling for 20,000 cycles. SBS was measured one-day post polymerization and after thermocycling using universal testing machine. SBS values and failure modes were recorded and statistically analyzed.

Results: the experimental group presented significantly lower resin-dentin SBS than the control group. Thermocycling significantly lowered SBS values for both groups. When using SE mode, the experimental group reported lower resin-dentin SBS than the control group before and after thermocyclying. When using ER mode before thermocycling, no significant difference was found between the two groups, while after thermocycling, the experimental group reported significantly higher resin-dentin SBS than the control group. For both adhesive groups, mixed failure dominated with ER mode while adhesive failure dominated with SE mode.
\end{abstract}

Conclusions: Addition of HA may provide resistance to degradation and offer a potential to improve resin-dentin bond stability against thermal changes when ER protocol is applied.

KEYWORDS: hydroxyapatite, dentin-bonding, universal-adhesive, etch\&rinse

* Postgraduate MS Resident, Division of Operative and Esthetic Dentistry, Faculty of Dentistry, Beirut Arab University, Lebanon (BDS, University of Bagdad, Iraq)

** Professor of Operative and Esthetic Dentistry, Faculty of Dentistry, Beirut Arab University, Lebanon (BDS, MSc, $\mathrm{PhD}$, Operative and Esthetics, Cairo University, Egypt)

*** Professor of Biomaterials, Faculty of Dentistry, Beirut Arab University, Lebanon (BDS, MSc, PhD, Dental Materials, Alexandia University, Egypt) 


\section{INTRODUCTION}

Universal adhesives (UA) are gaining wide acceptability among dentists due to their versatility of application and their capability to bond effectively to different substates including enamel, dentin, direct and indirect restorations. ${ }^{1}$

UAs constitute one type of all-in-one adhesive with both, hydrophilic and hydrophobic components in the same bottle. UAs contain monomer blends of mild to moderate acidity (phosphate, carboxylic etc.) in reduced concentrations compared with their precursors, ${ }^{2}$ conventional dimethacrylate cross-linkers, non-acidic emulsifying monomers, catalysts for light- or dual-curing and solvents to enhance monomer spreading and substrate infiltration capacity. Currently, monomers with mild acidity and water-insoluble salt formation capacity with dentin, such as 10-methacryloyloxydecyl dihydrogen phosphate (10-MDP), have been considered as the most reliable treatment for dentin and thus, it comprises the main monomer used in UAs. ${ }^{3}$ According to the manufacturers, UAs can be applied either by etch-and-rinse (ER) or the selfetch (SE) mode. ${ }^{4}$

Based on previous studies, when UAs were bonded to enamel using the SE mode, lower bond strength values were obtained than when using the ER mode. As a result, selective enamel etching has been suggested. The literature, on the other hand, is vague when it comes to dentin bonding. Even though sufficient resin-dentin bond strength can be achieved, lessened bonding efficiency arises with time and the stability of dentin-adhesive interface generated by these new multimode adhesive systems continue to be questionable. The in vitro evidence suggests that the application of UAs using some alternative techniques or additional strategies may be beneficial for improving their bonding performance to dentin.?

There are several attempts to replace water from resin-sparse regions of the hybrid layer with apatite crystallites that are sufficiently small to occupy the extra- and intrafibrillar compartments of the collagen matrix allowing biomineralization of the resin-dentin bond. ${ }^{8}$ These attempts were adopted by the concept of biomimetic mineralization that utilizes nano-technological principles to mimic natural biomineralization. ${ }^{9,10}$

Among the attempts to improve mechanical properties of dental adhesives and increase their bond strength to tooth tissues is the addition of bioactive hydroxyapatite (HA) nano-fillers. HA is a non-toxic biocompatible agent that has shown chemical stability in the physiological environment with specific biological actions. Its bioactivity and remineralization capability have enabled its use as filler in adhesives to enhance material properties. ${ }^{11}$

Initial studies on the incorporation of HA microfillers in adhesives presented improved bond strength and reduced degradation of the hybrid layer due to hydrophilic absorption property and enzymatic action. $^{12}$ Leitune and coauthors reported that inclusion of nano-HA particles in the experimental adhesive improved its bond strength to dentin. ${ }^{13}$ Moreover, it has been suggested that the use of nano-HA particles improves the surface area for adhesion and strengthens the mechanical properties of adhesive. ${ }^{14}$ Hoshika and co-workers speculated that incorporating HAp into dental adhesives may allow the formation of crystals below the hybrid layer, which could help create a stronger anchor. ${ }^{15}$ Lezaja et al suggested that HAP nano-fillers may improve bond strength of total-etch adhesives without an adverse effect on adhesive dispersion on dentin and its ability to infiltrate dentinal tubules. ${ }^{16}$

Until current date, there are limited reports available on the interaction of HA nanoparticles in universal adhesives. Moreover, no golden protocol for achieving stable and optimal adhesion of universal adhesive systems to dentin. ${ }^{7}$ This in vitro study aimed to evaluate the effect of addition of Hydroxyl-Apatite nanoparticles (HAp) 
to a commercial universal adhesive using two application modes, on SBS to dentin immediately and after thermocycling.

The null hypothesis was that there would be no difference in dentin-resin SBS between UA with and without the addition of HAp nanoparticles as well as between groups bonded with TE and SE application modes.

\section{MATERIALS AND METHODS}

This experimental in vitro study was conducted using 88 - freshly extracted sound caries-free human premolar teeth collected from orthodontic specialty clinic at Beirut Arab University (BAU) after the approval of the ethical committee of BAU. Teeth were disinfected and examined for any defects using magnifying loop (Eyemag pro $\mathrm{F}$ 4x/450 Carl ziess meditec AG, German), ultra-sonically cleaned and stored in $0.04 \%$ thymol at $4{ }^{\circ} \mathrm{C}$ until use. Materials used in the study are listed in table (1):

\section{Preparation of the experimental adhesive}

The experimental adhesive was prepared by adding $10 \%$ HAp (diameter under $20 \mathrm{~nm}$ ) to a commercial universal adhesive (Single bond universal, 3MESPE, Seefeld, Germany) as per
Hoshika et al. ${ }^{15}$ The HA particle size was under 20 $\mathrm{nm}$ to facilitate impregnation into the nanospaces within the hybrid zone. Three $\mathrm{mL}$ of UA was weighed on an analytical balance (Citizen ${ }^{\circledR}$ Digital Electronic Precision) accurately to $0.1 \mathrm{mg}$ in test tubes wrapped in tin foil to prevent light exposure. Based on the adhesive weight mass, the mass of HAp powder was calculated. Then the calculated amount of HAp powder was added to the adhesive. The mixture was manually shook for $5 \mathrm{~min}$ and used immediately after preparation. ${ }^{16}$

\section{Sample preparation and bonding}

Each tooth was embedded in acrylic resin to the enamel-cementum junction and sectioned perpendicular to the long axis to remove coronal enamel in order to facilitate locating the coronal dentin. Then the buccal enamel was removed to expose the mid-buccal dentin using a precision saw (IsoMet ${ }^{\circledR} 4000$ Linear Buehler ${ }^{\circledR}$, USA). The exposed buccal dentin was sandblasted with 600grit silicone paper (Buehler, Lake Bluff, IL, USA) under water irrigation to form a standard thickness of smear layer, then rinsed with distilled water for 10 seconds to remove the debris.

Samples were then allocated to 8 groups of 11 teeth per group. ER protocol was employed for

TABLE (1) Material description and manufacturer

\begin{tabular}{|c|c|c|}
\hline Material & Manufacturer & Composition \\
\hline $\begin{array}{l}\text { Single Bond } \\
\text { Universal }\end{array}$ & $\begin{array}{l}\text { 3M ESPE, Seefeld, } \\
\text { Germany }\end{array}$ & $\begin{array}{l}\text { 10-MDP phosphate monomer, BisGMA (15-25 wt } \%) \text {, HEMA }(15-25 \mathrm{wt} \%) \text {, } \\
\text { DMDMA (5-15 wt } \%) \text {, ethanol (10-15 wt } \%) \text {, water }(10-15 \mathrm{wt} \%) \text {, silica filler }(5-15 \\
\text { wt } \%), 2 \text {-propenoic acid }(1-10 \mathrm{wt} \%), 2 \text {-methyl-, reaction products with 1,10-decanediol } \\
\text { and phosphorous oxide (P2O5) (1-10 wt } \%) \text {, copolymer of acrylic itaconic acid }(1-5 \\
\mathrm{wt} \%) \text {, camphorquinone }(<2 \mathrm{wt} \%) \text {, silane, EDMAB }(2 \mathrm{wt} \%) \text {, toluene }(<0.02 \mathrm{wt} \%)\end{array}$ \\
\hline $\begin{array}{l}\text { Filtek }^{\mathrm{TM}} \\
\mathrm{Z350} \mathrm{XT}\end{array}$ & $\begin{array}{l}(3 \mathrm{M} \text { ESPE, } \\
\text { St.Paul, Minessota, } \\
\text { USA) }\end{array}$ & $\begin{array}{l}\text { Bis-GMA, UDMA,TEGDMA and BisEMA, Combination of aggregated zirconia/silica } \\
\text { cluster with primary particle size }(5-20 \mathrm{~nm}) \text {, and non-agglomerated silica filler (20 } \\
\mathrm{nm}) .78 .5 \mathrm{Wt} \%\end{array}$ \\
\hline HAp & (made in china) & $\begin{array}{l}\text { calcium phosphate (Hydroxyapatite; bone meal, nano-hydroxyapatite; HAP; CAS } \\
\text { number: (1306-06-5) }\end{array}$ \\
\hline
\end{tabular}


half of the experimental and the control adhesive groups while SE protocol was employed the other half. For ER protocol, dentin was etched by $37 \%$ phosphoric acid (N-Etch $®$, Ivoclar vivadent) for 15 seconds, washed with water-air jet and blot dried. The adhesive was applied in two layers using a microbrush, rubbed for 20 seconds and air blown for 5 seconds. For SE protocol, dentin was blot dried and the adhesive was applied in two layers, rubbed in for 20 seconds and air-blown for 5 seconds. For all groups, the adhesive was cured for 20 seconds using a LED light-curing unit.

A commercial nanohybrid composite (Filtek Z350 XT, 3M ESPE,St. Paul, MN, USA) was built-up in $1 \mathrm{~mm}$ increments over the polymerized adhesive using a customized split-mould with an internal diameter of $3 \mathrm{~mm}$ and height of $3 \mathrm{~mm}$ (ISO/ TS 11405:2003), and light cured for $40 \mathrm{sec}$.

\section{Storage}

Half the samples of each group were stored in distilled water for $24 \mathrm{~h}$ at $37{ }^{\circ} \mathrm{C}$ for immediate testing whereas the other half was subjected to thermocycling for 20,000 cycles (cyclic immersion for 30 sec. at $5.58 \mathrm{C} / 558 \mathrm{C}$ each, $7 \mathrm{sec}$. dwelling time). Based on the previous research every 5000 cycles correspond to six months of in vivo functioning. ${ }^{17}$

\section{Shear Bond Strength Testing:}

SBS was measured one-day post-polymerization using a universal testing machine (Instron Universal Testing Machine, England) with a knife-edge shearing blade at a crosshead speed of $1 \mathrm{~mm} / \mathrm{min}$ until fracture. A custom-made tooth holder made for tooth fixation. The shearing blade was pointed to the composite build-up parallel to the bonded surface and $1 \mathrm{~mm}$ away from the composite-dentin interface. Maximum force at fracture in Newton $(\mathrm{N})$ was recorded. SBS [MPa] to dentin was calculated dividing the maximum force at fracture $[\mathrm{N}]$ with the bonded surface area $\left[\mathrm{mm}^{2}\right]$.

\section{Failure Mode Evaluation}

After failure, dentin surfaces were assessed to determine the type of failure under a light microscope at x40 magnification. Failure modes were categorized into adhesive, cohesive and mixed types.

\section{Statistical Analysis}

The means and standard deviations of SBS were analyzed using ANOVA. When there is a significant difference between two groups t- test was used. Statistical analysis was performed with SPSS (statistical package for the social sciences) v.25 (IBM, New York, NY). Statistical significance level was established at $\mathrm{p}<0.05$.

\section{RESULTS}

\section{Resin-dentin SBS Results}

The study compared the resin-dentin SBS between the two adhesive groups, experimental and control adhesive, each categorized according to the etching mode (ER \& SE) and thermocycling.

Regardless of the etching mode and thermocycling, SBS of experimental adhesive group was significantly lower than the control adhesive group $(20.1 \pm 4.5$ and $23.5 \pm 4.3$ respectively, $\mathrm{p}<0.05)$.

Based on the etching mode, SBS of SE- experimental adhesive group- was significantly lower than the SE-control adhesive group $(\mathrm{p}<0.05)$ both, before and after thermocycling. SBS of ER- experimental adhesive group- was significantly lower than the ER-control adhesive group $(\mathrm{p}<0.05)$ before thermocycling $(26.1 \pm 1.34 \mathrm{MPa}, 28.43 \pm 2.68 \mathrm{MPa}$ respectively), whereas ER-experimental adhesive group had significantly higher mean SBS value than the ER-control adhesive group after thermocycling $(23.05 \pm 1.14 \mathrm{MPa}, 21.61 \pm 1.42 \mathrm{MPa}$ respectively) as presented in table $2 \&$ figure 1. 
TABLE (2) The mean, standard deviation (SD) values and results of independent samples t-test for the effect of adding HAp to UA on SBS based on etching mode and thermocycling

\begin{tabular}{|c|c|c|c|c|c|c|}
\hline \multirow{2}{*}{$\begin{array}{c}\text { Etching } \\
\text { Mode }\end{array}$} & \multirow{2}{*}{ Thermocycling } & \multicolumn{2}{|c|}{ HAp adhesive treated group } & \multicolumn{2}{c|}{ Without HAp adhesive treated group } & \multirow{2}{*}{ P-value } \\
\cline { 3 - 7 } & & Mean & SD & Mean & SD & \\
\hline \multirow{3}{*}{ Self-Etch } & Before & 17.17 & 1.48 & 25.43 & 1.62 & $0.000 *$ \\
\cline { 2 - 7 } & After & 15.13 & 1.11 & 18.48 & 1.94 & $0.000 *$ \\
\hline \multirow{2}{*}{ Total Etch } & Before & 26.1 & 1.34 & 28.43 & 2.68 & $0.024 *$ \\
\cline { 2 - 7 } & After & 23.05 & 1.14 & 21.61 & 1.42 & $0.022^{*}$ \\
\hline
\end{tabular}

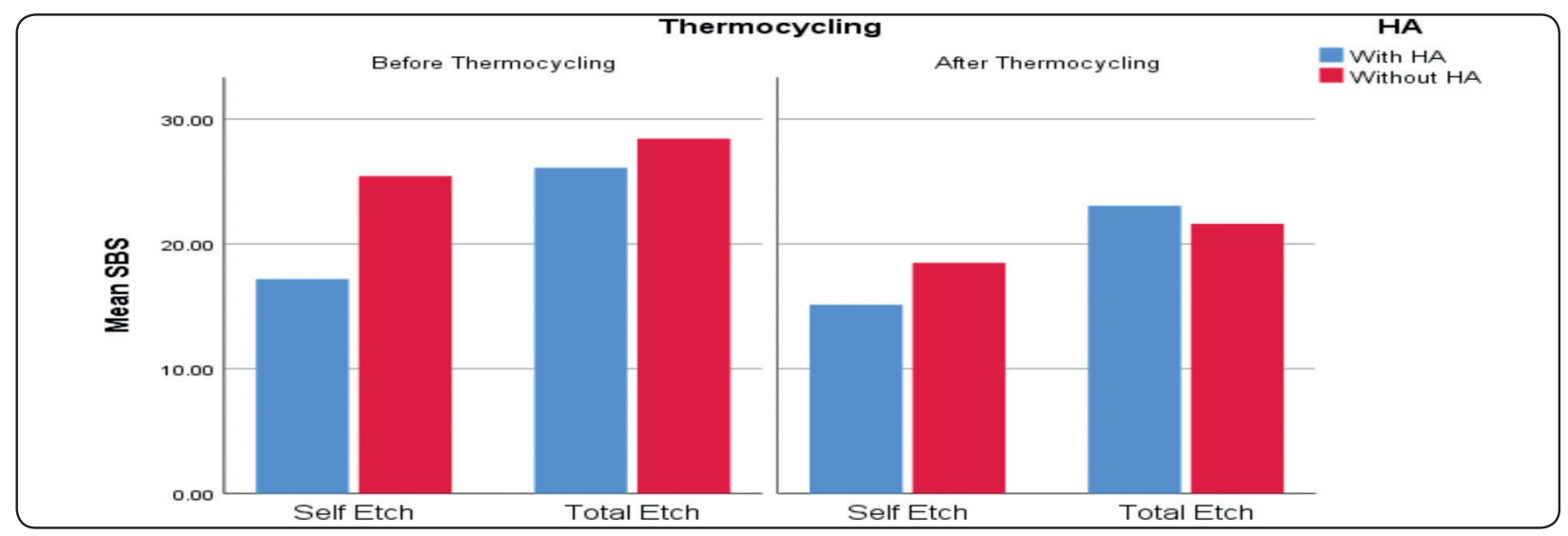

Fig. (1) Bar chart representing comparison between groups as mean values of SBS of independent samples based on etching mode and thermocycling

As to the overall effect, SE application mode significantly reduced SBS as compared to ER application mode $(19.1 \pm 4.2,24.5 \pm 3.3$ respectively, $\mathrm{p}<0.05)$. Additionally, both adhesives showed negative influence of thermocycling on SBS $(19.3 \pm 3.1,24.3 \pm 4.7, \mathrm{p}<0.05)$.

\section{Failure mode Results}

For both adhesive groups before and after thermocycling, adhesive failure was predominated when SE-was employed. While mixed failure was predominated when ER mode was employed. Table 3 , figure reveals details of the failure mode.

TABLE (3) Failure mode analysis

\begin{tabular}{|c|c|c|c|c|c|}
\hline Type of failure & & Adhesive $(\mathrm{n}=11)$ & Adhesive & Cohesive & Mixed \\
\hline \multirow{4}{*}{ Before thermocycling } & \multirow{2}{*}{ SE } & HA-Group & (11) 100 & 0 & 0 \\
\hline & & CA-Group & (8) 72.73 & 0 & (3) 27.27 \\
\hline & \multirow{2}{*}{ ER } & HA- Group & 0 & 0 & (11) 100 \\
\hline & & CA- Group & 0 & 0 & (11) 100 \\
\hline \multirow{4}{*}{ After thermocycling } & \multirow{2}{*}{$\mathrm{SE}$} & HA- Group & (11) 100 & 0 & 0 \\
\hline & & CA- Group & (11) 100 & 0 & 0 \\
\hline & \multirow{2}{*}{ ER } & HA- Group & (1) 9.09 & 0 & (10) 90.91 \\
\hline & & CA- Group & 0 & 0 & (11) 100 \\
\hline
\end{tabular}


After thermocycling, two representative SEM images of the experimental adhesive were captured. With SE mode, HA agglomerates were observed in the adhesive layer. No hybrid layer detected (figure 2). With ER mode, HA agglomerates demonstrated clustering above hybrid layer (figure 3).

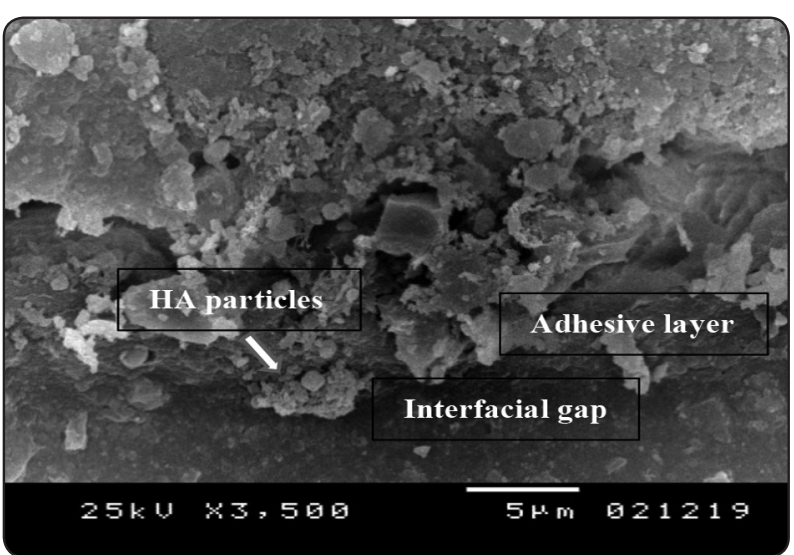

Fig. (2) SEM image at $3500 \mathrm{x}$ of experimental adhesive in SE mode demonstrating presence of HA particles agglomerate in the adhesive resin. No hybrid layer was observed.

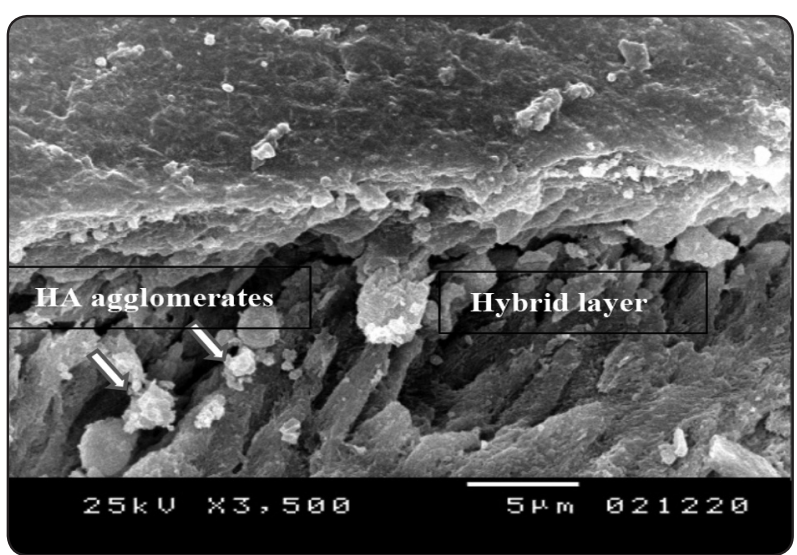

Fig. (3) SEM image at $3500 \mathrm{x}$, of experimental adhesive in ER mode demonstrating clustering of HA agglomerates above hybrid layer. Hybrid layer with resin tags is observed.

\section{DISCUSSION}

Despite the numerous studies that have shown the bonding effectiveness of the newest class of bonding agents "the universal adhesives", there are still some unresolved problems regarding the durability of the resin-dentin interface. ${ }^{7}$ Moreover, there is limited information as to whether the different etching modes achieve equivalent bonding performance to dentin when it is subjected to aging. ${ }^{18}$ In order to achieve resin-dentin interface that is less susceptible to degradation, studies have proposed incorporation of nanofillers to reduce hydrophilicity, minimize enzymatic collagen degradation, and lower stress contraction relatively due to a decrease in a polymeric matrix. ${ }^{19}$ It was speculated that incorporation of HA nano particles in resin adhesives can interact within the hybrid layer, occupy the extra and intrafibrillar compartments of the collagen matrix, replace water with apatite crystallites and form an organic bond that contribute positively to its bond strength to tooth..$^{20}$

A commercial universal adhesive (Single Bond Universal) was selected for this as a control adhesive. Previous studies reported more stable dentinresin interface under different moisture situations when compared to other universal adhesives and accredited their results to its unique chemistry. ${ }^{21,22}$ The same commercial adhesive was modified by preparing and adding 10 wt.\% HA nanospheres, less than $20 \mathrm{~nm}$ in diameter and considered as experimental adhesive. This percentage was used based on the confirmation of a previous research that $10 \%$ HA produced fast growth of large crystal when compared to $5 \%$ HA. ${ }^{15}$ The HA particle size was under $20 \mathrm{~nm}$ to facilitate impregnation into the nanospaces within the hybrid zone.

The results of our study revealed that SBS of the experimental group was inferior as compared to the control group. One possible explanation could be related to the increased viscosity of the experimental adhesive which may compromise the wetting of dentin and adversely affect shear 
bond strength. ${ }^{18}$ Adding $10 \%$ HAp may occupy the water-filled spaces in the demineralized dentin, and may interfere with resin infiltration. It's worth mentioning that the adhesive used contain 10-MDP monomer (Single-Bond Universal). 10-MDP monomer from SBU was previously shown to interact chemically with partially demineralized HA in dentin and reported to strengthen the mechanism of bonding. ${ }^{16}$ It has not been investigated if 10-MDP monomer would potentially interact with HAp fillers in the adhesive and if this interaction would disperse the adhesive on dentin or buffer the acidity of the adhesive. ${ }^{25}$ All these postulations may contribute to the inferior SBS of the experimental adhesive. Although some studies suggested that addition of fillers into adhesive systems wouldn't affect the bond strength, ${ }^{25}$ more recent studies reported increased bond strength when HA fillers were added to the adhesives. ${ }^{11}$ They accredited their results to the methodology they used to prepare HA containing adhesives. In fact, they silanized the commercial HAp using 5\% silane to improve adhesion to the resin matrix and used 5wt \% HA. In our study, we added 10 wt $\%$ non silanized HA as the commercial adhesive used already contain silane. We anticipated that adding silane would hinder the reactivity of HA.

Regardless of the adhesive type or thermocycling, employing ER mode showed superior SBS results as compared to SE mode. Using universal adhesives in $\mathrm{SE}$ mode limit the demineralization of dentin to leave some minerals for chemical bonding. ${ }^{34}$ However, the hydrophilic nature of dentin as well as the hydrophilicity of the adhesive may lead to increase the water content in the adhesive layer which might impair polymerization and decrease bond strength to dentin. ${ }^{27}$ On the other hand, ER mode, remove smear layer, demineralize few microns of dentin layer and allow for resin impregnation and form micromechanical interlocking. SBS values of RE adhesives were found to be greater than SE adhesives by many researchers. ${ }^{28}$ Some studies that used phosphoric acid etching prior to onestep self-etch adhesive and showed increased in
SBS. ${ }^{29}$ On contrast, other studies, stated that the SBS showed no significant effect to dentin after using phosphoric acid etch prior to one-step selfetch adhesive. ${ }^{30}$ It seems that the controversy in the literature is related to the difference the composition between the adhesives tested. A recent literature review suggested that better bond stability with SE adhesives would be obtained when the application procedures are modified. ${ }^{7}$

When SE mode was employed, there was a significant decrease in SBS of the experimental adhesive as compared to the control adhesive before and after thermocycling. This could be related to the interaction of HAp with the acidic monomers, buffering their acidity, leaving dentin with minimal demineralization and thus, no space for the resin to penetrate and hybridize within dentin. Although, chemical bonding with dentin hydroxyapatite is anticipated yet, this bond was not strong enough to resist shear forces as the micromechanical bond that is created by ER protocol. The phosphoric acid strong acidity, on the other hand, would allow for adequate dentin demineralization and, as a result, deep hybridization of the resin into dentin. The resin's failure to impregnate to the maximum extent of the hybrid layer, which challenges hydrolytic degradation, is one potential drawback.

Although the degradation mechanism is not well known, increased enzymatic activity, elution and plasticization of monomers by water are some of the contributing factors. ${ }^{42}$ Addition of HA may provide resistance to degradation as they interact with water, form apatite crystals and enhance the remineralization process. The minerals in the experimental adhesive may act as a constant site for backup minerals that induce continuous remineralization for a period of time. ${ }^{32}$ This may explain why the experimental adhesive's SBS was significantly higher than the control adhesive when the ER mode was used after thermocycling. As a result, the current findings indicate that adding HAp to the adhesive may improve resin bond strength to dentin when ER mode is employed. 
Thermocycling significantly decreased SBS of both adhesive groups. These results are in line with many previous investigations that verified the deleterious effect of thermal changes on the integrity of resin-adhesive interface. ${ }^{4,33}$

A plausible finding in this study is that HAcontaining adhesive resin demonstrated more resistance to degradation than the control. This was noticed when comparing the mean values of SBS before and after thermocycling of each group. After thermocycling, the drop of SBS values of HA-containing adhesive resin was significantly low compared to the control. The precise structure of the HA nanoparticles may alter the mechanical properties through interaction with the dense collagen network. This complex interaction is supported by the hydrogen bonding between $\mathrm{COOH},-\mathrm{OH}$, and $-\mathrm{NH} 2$ of the collagen network with the $-\mathrm{OH}$ group of the hydroxyapatite particles, triggering re-mineralization at the interface of adhesive dentin. ${ }^{34}$ Additionally, HA particles may act as centres for chemical interaction with acidic monomers such as 10-MDP.

It's worth pointing out that the average dentin macro-shear bond strength values for the two adhesives used were in the range of $13.8-32.8 \mathrm{MPa}$ which correspond well with the average macroshear bond strength values for majority of adhesives which were in the range of 10-20 MPa as provided from a literature review and meta-analysis by De Munck et al. ${ }^{35}$

The adhesive layer is the weakest area of the interface between the tooth and restoration, which causes a greater stress concentration by load. ${ }^{36}$ In this study, the failure mode was either adhesive or mixed failure. The type of failure was not affected by the presence of HAP particles but was dependent on the etching mode and thermocycling i.e. samples with ER protocol showed higher SBS and tended to have more 'mixed' failures i.e. combination of adhesive and cohesive failure. While samples with SE protocol showed lower SBS and tended to have more "adhesive failures". Additionally, after thermocycling, $100 \%$ of the samples showed an adhesive failure. These findings were in agreement with several studies. . $^{16,37,38}$

Since there was a significant difference in SBS between the experimental and control groups, the null hypothesis was rejected except for the ER protocol before thermocycling, the null hypothesis was accepted.

One of the limitations of this study is that there was little knowledge in the literature at the time of this investigation about the optimum level of HAp to be incorporated in adhesives. In future studies, the HAp concentration chosen for this analysis may be reduced in order to decrease the viscosity of the adhesive. Additionally, to strengthen HA binding to the resin matrix, silanization of the HA fillers could be suggested. Future researches shall consider adding HA fillers to the adhesive systems and investigate their effect on durability of the bonded interface.

\section{CONCLUSIONS}

Within the limitations of this study:

1. HAp modified UA showed inferior resin-dentin SBS compared to the control adhesive.

2. Resin-dentin SBS was affected by the application protocol and thermocyling rather than the presence or absence of HAp.

3. ER protocol presented superior resin-dentin SBS results compared to SE protocol.

4. HA-modified universal adhesives may offer resistance to degradation.

5. There is a potential in HAp modified adhesive to improve resin-dentin bond stability against thermal changes when ER protocol is applied. 


\section{REFERENCES}

1. Cuevas-Suárez, C. E., de Oliveira da Rosa, W. L., Vitti, R. P., da Silva, A. F., \& Piva, E. (2020). Bonding Strength of Universal Adhesives to Indirect Substrates: A Meta-Analysis of in Vitro Studies. Journal of prosthodontics, 29(4), 298-308.

2. Chen, C., Niu, L. N., Xie, H., Zhang, Z. Y., Zhou, L. Q., Jiao, K., ... \& Tay, F. R. (2015). Bonding of universal adhesives to dentine-Old wine in new bottles. Journal of dentistry, 43(5), 525-536.

3. Carrilho, E., Cardoso, M., Marques Ferreira, M., Marto, C. M., Paula, A., \& Coelho, A. S. (2019). 10-MDP based dental adhesives: Adhesive interface characterization and adhesive stability-A systematic review. Materials journal, 12(5), 790 .

4. Papadogiannis, D., Dimitriadi, M., Zafiropoulou, M., Gaintantzopoulou, M. D., \& Eliades, G. (2019). Universal adhesives: setting characteristics and reactivity with dentin. Materials journal, 12(10), 1720.

5. Nagarkar, S., Theis-Mahon, N., \& Perdigão, J. (2019). Universal dental adhesives: Current status, laboratory testing, and clinical performance. Journal of Biomedical Materials Research Part B: Applied Biomaterials journal, 107(6), 2121-2131.

6. Ahmed, M. H., Yoshihara, K., Mercelis, B., Van Landuyt, K., Peumans, M., \& Van Meerbeek, B. (2019). Quick bonding using a universal adhesive. journal of clinical oral investigations, 1-15.

7. Hardan, L., Bourgi, R., Kharouf, N., Mancino, D., Zarow, M., Jakubowicz, N., ... \& Cuevas-Suárez, C. E. (2021). Bond Strength of Universal Adhesives to Dentin: A Systematic Review and Meta-Analysis. Polymers journal, 13(5), 814.

8. Yoshihara, K., Yoshida, Y., Hayakawa, S., Nagaoka, N., Irie, M., Ogawa, T., ... \& Van Meerbeek, B. (2011). Nanolayering of phosphoric acid ester monomer on enamel and dentin. Acta biomaterialia journal, 7(8), 3187-3195.

9. Tay, F. R., \& Pashley, D. H. (2008). Guided tissue remineralisation of partially demineralised human dentine. Biomaterials journal, 29(8), 1127-1137.

10. Frassetto, A., Breschi, L., Turco, G., Marchesi, G., Di Lenarda, R., Tay, F. R., \& Cadenaro, M. (2016). Mechanisms of degradation of the hybrid layer in adhesive dentistry and therapeutic agents to improve bond durability - A literature review. Dental materials journal, 32(2), e41-e53.
11. Al-Hamdan, R. S., Almutairi, B., Kattan, H. F., Alresayes, S., Abduljabbar, T., \& Vohra, F. (2020). Assessment of hydroxyapatite nanospheres incorporated dentin adhesive. A SEM/EDX, micro-raman, microtensile and micro-indentation study. Coatings journal, 10(12), 1181.

12. Sadat-Shojai, M., Atai, M., Nodehi, A., \& Khanlar, L. N. (2010). Hydroxyapatite nanorods as novel fillers for improving the properties of dental adhesives: Synthesis and application. Dental materials journal, 26(5), 471-482.

13. Leitune, V. C. B., Collares, F. M., Trommer, R. M., Andrioli, D. G., Bergmann, C. P., \& Samuel, S. M. W. (2013). The addition of nanostructured hydroxyapatite to an experimental adhesive resin. Journal of dentistry, 41(4), 321-327.

14. Melo, M. A. S., Cheng, L., Zhang, K., Weir, M. D., Rodrigues, L. K., \& Xu, H. H. (2013). Novel dental adhesives containing nanoparticles of silver and amorphous calcium phosphate. Dental materials journal, 29(2), 199-210.

15. Hoshika, T., Nishitani, Y., Takahashi, K., Ogata, H., Ohara, N., Kajihara, T., \& Yoshiyama, M. (2015). Mineralization of resin using experimental adhesives containing hydroxyapatite in long term. Journal of oral tissue engineering, 13(2), 85-95.

16. Lezaja, M., Jokic, B. M., Veljovic, D. N., \& Miletic, V. (2016). Shear bond strength to dentine of dental adhesives containing hydroxyapatite nano-fillers. Journal of adhesion science and technology, 30(24), 2678-2689.

17. Gale, M. S., \& Darvell, B.W. (1999). Thermal cycling procedures for laboratory testing of dental restorations. Journal of dentistry, 27(2), 89-99.

18. Jayasheel, A., Niranjan, N., Pamidi, H., \& Suryakanth, M. B. (2017). Comparative evaluation of shear bond strength of universal dental adhesives-an in vitro study. Journal of clinical and experimental dentistry, 9(7), e892.

19. Makvandi, P., Gu, J. T., Zare, E. N., Ashtari, B., Moeini, A., Tay, F. R., \& Niu, L. N. (2020). Polymeric and inorganic nanoscopical antimicrobial fillers in dentistry. Acta biomaterialia journal, 101, 69-101.

20. Niu, L. N., Zhang, W., Pashley, D. H., Breschi, L., Mao, J., Chen, J. H., \& Tay, F. R. (2014). Biomimetic remineralization of dentin. Dental materials journal, 30(1), 77-96.

21. Mena-Serrano, A., Kose, C., De Paula, E. A., Tay, L. Y., Reis, A., Loguercio, A. D., \& Perdigão, J. (2013). A new universal simplified adhesive: 6-month clinical evaluation. Journal of Esthetic and Restorative Dentistry, 25(1), 55-69. 
22. Shirban, F., Khoroushi, M., \& Shirban, M. (2013). A new solvent-free one-step self-etch adhesive: Bond strength to tooth structures. The journal of contemporary dental practice, 14(2), 269.

23. Nunes, M. F., Swift, E. J., \& Perdigao, J. (2001). Effects of adhesive composition on microtensile bond strength to human dentin. American journal of dentistry, 14(6), 340-343.

24. Yoshida, Y., Nagakane, K., Fukuda, R., Nakayama, Y., Okazaki, M., Shintani, H., \& Van Meerbeek, B. (2004). Comparative study on adhesive performance of functional monomers. Journal of dental research, 83(6), 454-458.

25. Lee, Y., Pinzon, L. M., O Keefe, K. L., \& Powers, J. M. (2006). Effect of filler addition on the bonding parameters of dentin bonding adhesives bonded to human dentin. American journal of dentistry, 19(1), 23.

26. Yoshihara, K., Yoshida, Y., Hayakawa, S., Nagaoka, N., Irie, M., Ogawa, T., \& Van Meerbeek, B. (2011). Nanolayering of phosphoric acid ester monomer on enamel and dentin. Acta Biomaterialia journal, 7(8), 3187-3195.

27. Miletic, V., Pongprueksa, P., De Munck, J., Brooks, N. R., \& Van Meerbeek, B. (2013). Monomer-to-polymer conversion and micro-tensile bond strength to dentine of experimental and commercial adhesives containing diphenyl (2, 4, 6-trimethylbenzoyl) phosphine oxide or a camphorquinone/amine photo-initiator system. Journal of dentistry, 41(10), 918-926.

28. Muñoz, M. A., Luque, I., Hass, V., Reis, A., Loguercio, A. D., \& Bombarda, N. H. C. (2013). Immediate bonding properties of universal adhesives to dentine. Journal of dentistry, 41(5), 404-411.

29. Alqahtani, M. Q. (2015). Influence of acid-etching or double-curing time on dentin bond strength of one-step selfetch adhesive. The Saudi journal for dental research, 6(2), 110-116.

30. Sabatini, C., Patel, M., \& D'Silva, E. (2013). In vitro shear bond strength of three self-adhesive resin cements and a resin-modified glass ionomer cement to various prosthodontic substrates. Operative dentistry journal, 38(2), 186196.

31. Tay, F. R., \& Pashley, D. H. (2003). Water treeing--a potential mechanism for degradation of dentin adhesives. American journal of dentistry, 16(1), 6-12.

32. Gandolfi, M. G., Taddei, P., Tinti, A., Dorigo, E. D. S., \& Prati, C. (2011). Alpha-TCP improves the apatite-formation ability of calcium-silicate hydraulic cement soaked in phosphate solutions. Materials Science and Engineering journal, 31(7), 1412-1422.

33. Hu, X., Luong, M. N., Zhang, H., Zhu, H., Chan, D. C., \& Sadr, A. (2019). Influence of phosphoric acid etching on the dentin bond durability of universal adhesives. Journal of adhesion science and technology, 33(21), 2356-2368.

34. Erol-Taygun, M., Unalan, I., Idris, M. I. B., Mano, J. F., \& Boccaccini, A. R. (2019). Bioactıve glass-polymer nanocomposites for bone tıssue regeneration applications: A review. Advanced engineering materials journal, 21(8), 1900287.

35. De Munck, J., Mine, A., Poitevin, A., Van Ende, A., Cardoso, M. V., Van Landuyt, K. L., \& Van Meerbeek, B. (2012). Meta-analytical review of parameters involved in dentin bonding. Journal of dental research, 91(4), 351-357.

36. Armstrong, S., Geraldeli, S., Maia, R., Raposo, L. H. A., Soares, C. J., \& Yamagawa, J. (2010). Adhesion to tooth structure: a critical review of "micro" bond strength test methods. Dental materials journal, 26(2), e50-e62.

37. Wagner, A., Wendler, M., Petschelt, A., Belli, R., \& Lohbauer, U. (2014). Bonding performance of universal adhesives in different etching modes. Journal of dentistry, 42(7), 800-807.

38. Moritake, N., Takamizawa, T., Ishii, R., Tsujimoto, A., Barkmeier, W. W., Latta, M. A., \& Miyazaki, M. (2019). Effect of active application on bond durability of universal adhesives. Operative dentistry journal, 44(2), 188-199. 